\title{
Urogenital Tuberculosis: Patient Classification in Seven Different Groups According to Clinical and Radiological Presentation
}

\author{
Andre A. Figueiredo, Antonio M. Lucon, Cristiano M. Gomes, Miguel Srougi \\ Division of Urology, University of Sao Paulo School of Medicine, Sao Paulo, Brazil
}

\begin{abstract}
Purpose: To describe and classify 80 cases of urogenital tuberculosis in seven groups of similar clinical and radiological presentation.

Materials and Methods: 80 patients (56 males, 70\%; median age 34 years; age range 12 to 75 ) with urogenital tuberculosis were retrospectively reviewed. The patients were divided in seven groups: 1) Bilateral parenchymatous renal lesions; 2) No or minimal changes on radiographic examination; 3) Unilateral renal tuberculosis; 4) Contracted bladder; 5) Contracted bladder with renal failure; 6) Tuberculosis on a transplanted kidney; 7) Isolated genital tuberculosis.

Results: 1) Seven (8.8\%) patients had multiple bilateral parenchymatous renal lesions with fever and malaise, characteristic of miliary tuberculosis. Three of these patients had AIDS. 2) Six (7.5\%) cases had an early diagnosis, with minimal or no radiographic lesions. Two did not have any urologic symptoms. 3) Twelve (15\%) patients had unilateral renal tuberculosis with partial (1 case) or total non-function kidney. 4) Thirty-seven (46.3\%) patients had contracted bladder associated with unilateral partial (1 case) or total non-function kidney. 5) Ten (12.5\%) patients had end stage renal disease due to tuberculosis with contracted bladder. 6) Four (5.0\%) patients had tuberculosis on a transplanted kidney, with graft loss in half the cases. 7) Four (5.0\%) patients had prostate or epididymis tuberculosis without associated renal lesion.

Conclusions: Urogenital tuberculosis is a destructive disease of the urogenital tract with variable clinical and radiographic presentation. A classification according to similar patterns correlating with disease stage is feasible although early diagnosis is the only prevention of the most severe forms.
\end{abstract}

Key words: kidney; ureter; bladder; tuberculosis

Int Braz J Urol. 2008; 34: 422-32

\section{INTRODUCTION}

Tuberculosis is a worldwide disease with greater prevalence wherever the population is concentrated in areas with poor sanitation and unfavorable social and economic indicators. Thirty percent of the world's population ( 1.7 billion people) is estimated to harbor the latent form of Mycobacterium tuberculosis (1-3). In spite of an effective pharmacological treatment and other technological breakthroughs, recent years have witnessed the recrudescence of the infec- tion, due to the appearance of drug-resistant bacilli, population migrations, and the AIDS epidemic (4). Only 22 countries concentrate $80 \%$ of the annual cases, with Brazil (80 to 90 thousand new cases a year since 1980) being one of them (5).

From the pulmonary focus, 2 to $20 \%$ of the patients go on to develop urogenital tuberculosis. Through hematogenous spread to the kidneys, prostate, and epididymis, through a descending route to the ureters, bladder, and urethra, and through the canalicular route to the genital organs $(3,4)$. Urogenital 
tuberculosis affects all age ranges, with predominance of 30 to 50 -year-old males $(6,7)$. Because of its insidious evolution and late-onset symptoms, diagnosis and treatment are delayed, with a consequent high rate of urogenital organ destruction and renal failure (8).

As all urogenital organs may be involved, tuberculosis may give rise to all urologic symptoms, adding to the complexity of the clinical and radiographic pictures. We attempted to classify patients with urogenital tuberculosis into identifiable groups with similar clinical and radiographic features.

\section{MATERIALS AND METHODS}

Eighty patients with urogenital tuberculosis, seen during the 1989-2005 period were retrospectively reviewed. These patients were treated in our tertiary teaching hospital that provides medical assistance free of charge to the metropolitan Sao Paulo area. Most of them have poor socio-economic conditions. There were $56(70 \%)$ males and $24(30 \%)$ females with a median age of 34 years (12 to 75 ). Figure-1 shows the distributions according to the decades. Urogenital tuberculosis was diagnosed by direct bacilli identification or culture growth in the urine of 36 (45 $\%)$ patients; histopathology in $25(31.3 \%)$ patients; and a combination of strong clinical, laboratory, and radiographic evidence of urogenital tuberculosis with negative bacilli search in the urine of $19(23.7 \%)$ patients.

The clinical features and the organs involved were described. The patients were classified in seven groups according to their patterns of initial clinical and radiographic presentation: 1) Bilateral parenchymatous renal lesions; 2) No or minimal changes on radiographic examination; 3) Unilateral renal tuberculosis; 4) Contracted bladder; 5) Contracted bladder with renal failure; 6) Tuberculosis on a transplanted kidney; 7) Isolated genital tuberculosis.

\section{RESULTS}

Table- 1 shows details about the signs and symptoms. Storage symptoms and hematuria were the most frequent symptoms, being present in $72.5 \%$ and $56.3 \%$ of the patients, respectively. Sixteen patients had some form of immunodeficiency: four because of AIDS, four with a kidney transplant and ongoing immunosuppressive therapy, four with diabetes, and four with alcohol abuse. In 35 (43.8\%) patients, there was clinical or radiographic evidence of previous tuberculosis. Table-2 shows the distribution of organ involvement. After tuberculosis diagnosis, all the patients received triple therapy for at least six months.

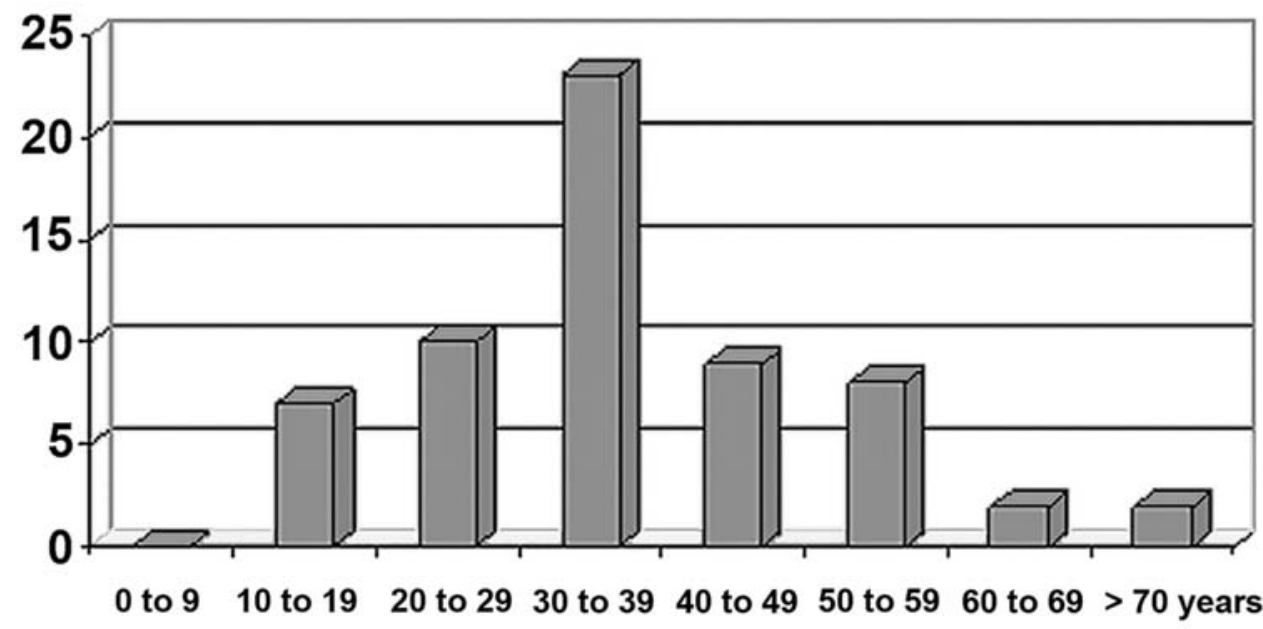

Figure 1 -Age distribution of the 80 patients with urogenital tuberculosis according to the decades. 
Table 1 - Presenting signs and symptoms in 80 patients with urogenital tuberculosis.

\begin{tabular}{lrr}
\hline Signs and Symptoms & N & \multicolumn{1}{c}{$\%$} \\
\hline Storage symptoms & 58 & $72.5 \%$ \\
Hematuria & 45 & $56.3 \%$ \\
Urinary tract infection & 14 & $17.5 \%$ \\
Lumbar pain & 23 & $28.8 \%$ \\
Perineal pain & 2 & $2.5 \%$ \\
Scrotal pain & 11 & $13.8 \%$ \\
Scrotal mass & 10 & $12.5 \%$ \\
Scrotal fistula & 4 & $5.0 \%$ \\
LUTS & 7 & $8.8 \%$ \\
Urinary retention & 3 & $3.8 \%$ \\
Fever, weight loss and asthenia & 36 & $45.0 \%$ \\
Urethral fistula & 1 & $1.3 \%$ \\
Renal failure & 10 & $12.5 \%$ \\
No symptoms from urinary tract & 5 & $6.3 \%$ \\
\hline
\end{tabular}

LUTS $=$ lower urinary tract symptoms.

Initial clinical and radiographic assessment yielded the following patterns of urogenital tuberculosis presentation:

1) Bilateral parenchymatous renal lesions - In seven $(8.8 \%)$ cases there were multiple bilateral parenchymatous renal lesions (Figure-2). In all cases, there were tuberculosis foci in other organs, fever, and malaise, characteristic of miliary tuberculosis. Three patients had AIDS. Case-fatality was $42.9 \%$ (three cases). The latter cases had the urogenital lesions diagnosed at postmortem examination. The other cases were diagnosed on imaging procedures. Three patients had no urologic symptom.

2) No or minimal changes on radiographic examination - In six (7.5\%) cases no lesions were found (four patients) or there were minimal lesions (two patients) on radiographic examination with unilateral renal calcification or calyx deformities (Figure-3). Tuberculosis was diagnosed when bacilli were shown in the urine. Four patients had hematuria and the other two had no urologic symptoms but the investigation was undertaken because of previous history of pulmonary tuberculosis. These patients did not require surgery and resolved well with pharmacological treatment alone.
3) Unilateral renal tuberculosis - In $12(15 \%)$ cases there was unilateral renal tuberculosis with obstruction and dilatation of the collecting system due to stenosis (Figure-4). In one case, there was single inferior pole function loss due to infundibular stenosis, which was treated with inferior polar nephrectomy. All the other cases underwent nephrectomy due to a non-functional kidney. In all cases, the contralateral kidney was normal on radiographic examination.

4) Contracted bladder - In 37 (46.3\%) patients, there was contracted bladder due to tuberculosis. All the cases had unilateral non-function kidney with dilatation of the collecting system due to stenosis, associated with a contracted bladder, except for one case with polar renal function loss. Voiding cystourethrogram showed no vesicoureteral reflux in 10 cases, bilateral reflux in two cases, and unilateral reflux to the contralateral functional kidney in 25 . The latter was the most frequent radiographic finding (Figure-5

Table 2 - Description of the affected organs.

\begin{tabular}{lrr}
\hline Affected Organ & N & \multicolumn{1}{c}{$\%$} \\
\hline Kidney & 72 & $90.0 \%$ \\
$\quad$ Bilateral and multiple lesions & 7 & $8.8 \%$ \\
Minimal lesions & 2 & $2.5 \%$ \\
Unilateral with polar loss of function & 2 & $2.5 \%$ \\
Unilateral with loss of function & 47 & $58.8 \%$ \\
Renal failure & 10 & $12.5 \%$ \\
Transplanted kidney & 4 & $5.0 \%$ \\
Ureter & 57 & $71.3 \%$ \\
& & \\
Bladder & 47 & $58.8 \%$ \\
Prostate & & \\
Prostate abscess & 6 & $7.5 \%$ \\
Prostatitis with perineal pain & 2 & $2.5 \%$ \\
No symptoms & 2 & $2.5 \%$ \\
& 2 & $2.5 \%$ \\
Epididymis & & \\
Unilateral & 10 & $12.5 \%$ \\
Bilateral & 8 & $10.0 \%$ \\
Cutaneous fistula & 2 & $2.5 \%$ \\
Epididymectomy & 4 & $5.0 \%$ \\
Seminal vesicles & 5 & $6.3 \%$ \\
\hline & 2 & $2.5 \%$ \\
\hline
\end{tabular}




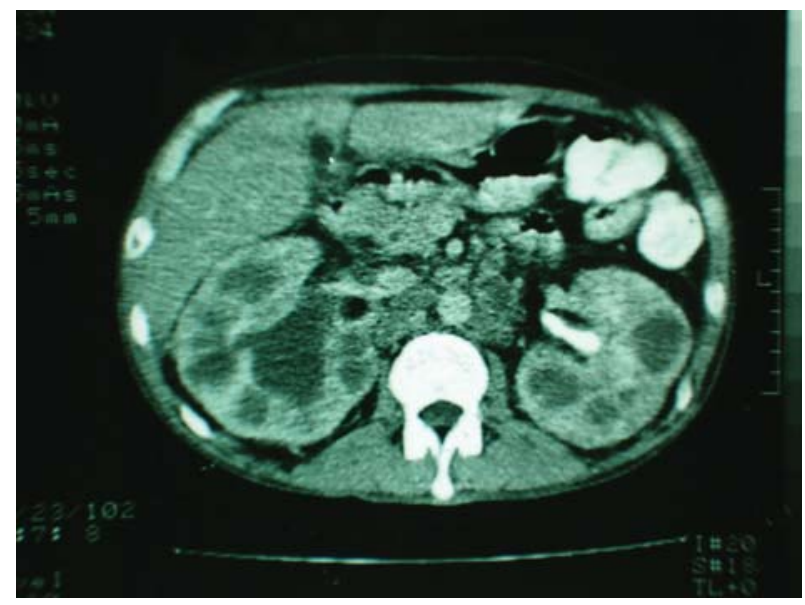

Figure 2-Computed tomography showing multiple and bilateral kidney lesions in a patient with miliary tuberculosis.

and 6). On radiographic examination, the functionally preserved contralateral kidney was normal in 23 cases and with ureterohydronephrosis in 14 , one of which showing areas of cortical retraction. In all these cases with ureterohydronephrosis, vesicoureteral reflux to the functionally preserved kidney was observed. The patients underwent bladder augmentation along with total nephrectomy of the non-function kidneys and partial nephrectomy in the case with polar disease. In the cases with high-degree reflux, the ureters were reimplanted. The patients did well but five progressed to chronic renal failure.

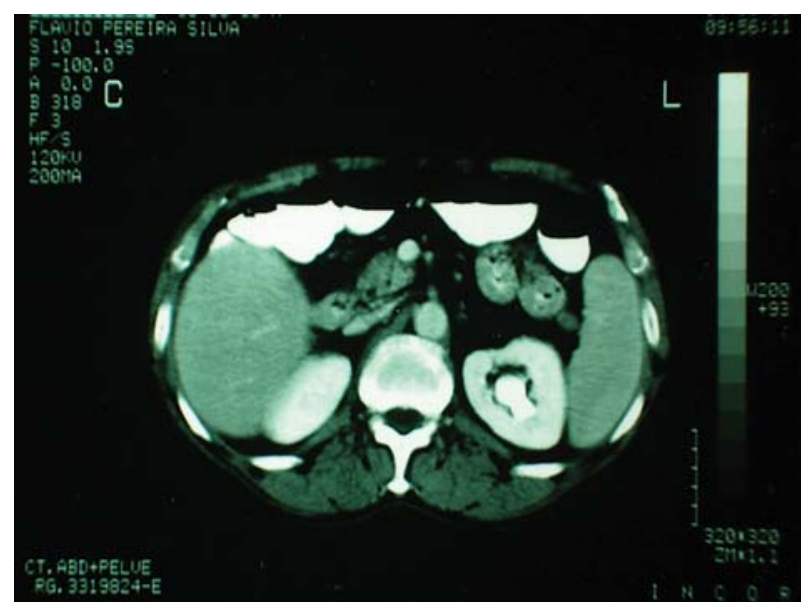

Figure 3 - Computed tomography showing calyceal dilatation in the left kidney.
5) Contracted bladder and renal failure - In 10 $(12.5 \%)$ cases the patients had end-stage renal disease due to tuberculosis. All had contracted bladder and unilateral vesicoureteral reflux. The patients underwent bilateral nephrectomy, bladder augmentation, and renal transplantation.

6) Tuberculosis on a transplanted kidney - In four $(5 \%)$ cases parenchymatous renal tuberculosis was diagnosed on a transplanted kidney. The patients had creatinine elevation and tuberculosis was diagnosed through biopsy (3 cases) or a finding of bacilli in the urine. There was graft loss in $50 \%$ of the cases.

7) Isolated genital tuberculosis - In four (5\%) cases there was genital tuberculosis without detectable
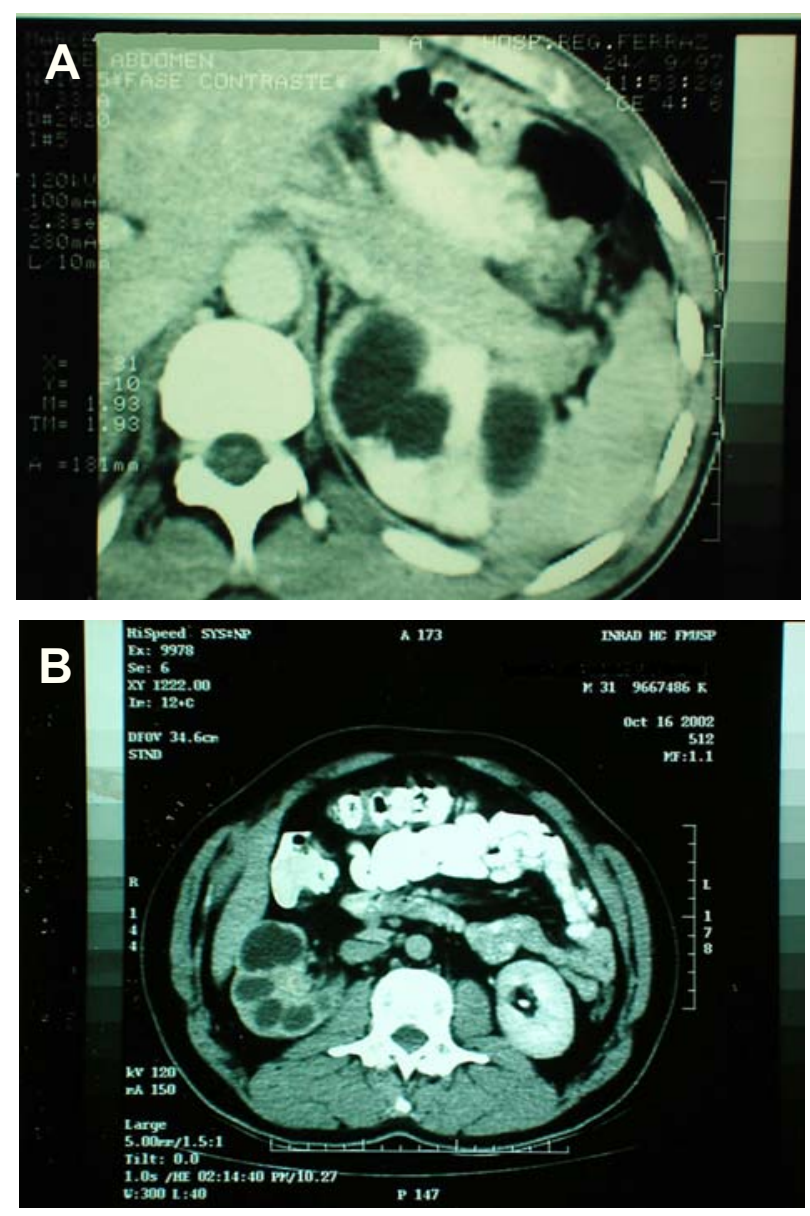

Figure 4-A) and B) Computed tomography showing unilateral renal tuberculosis with low function kidney with dilatation of the excretory system in two different patients. 

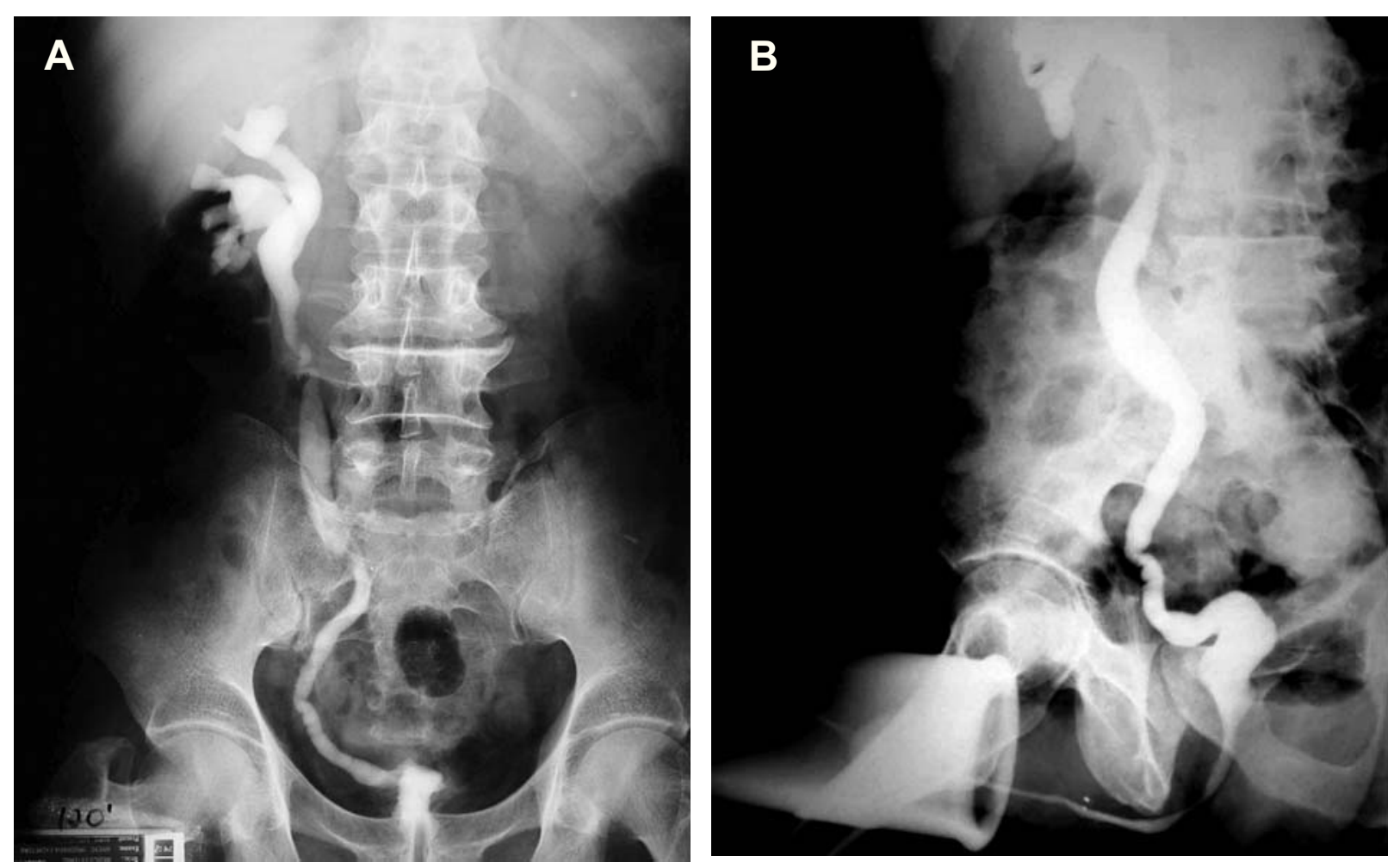

Figure 5 - A) Intravenous urography with left non-function kidney, B) Voiding cystography shows contracted bladder and right vesicoureteral reflux.

renal lesion. Two patients presented with prostate abscess and two with tuberculosis of the epididymis.

Tuberculosis of the epididymis also occurred in another eight cases with associated renal lesion, with a total of $10(12.5 \%)$ patients with pain and a mass over the epididymis, bilateral in two, and with cutaneous fistulization of an epididymis abscess in four. Because of extensive involvement, orchi-epididymectomy was necessary in $50 \%$ of the cases. Besides the two patients with prostate abscess, another four also had prostate tuberculosis. While two had storage symptoms and perineal pain, compatible with chronic prostatitis, two were asymptomatic and were serendipitously diagnosed by histopathology. Tuberculosis of the seminal vesicles occurred in two cases, one of which in the patient with prostate abscess and another as a histopathological finding after radical prostatectomy.

Of the 76 patients without tuberculosis on the transplanted kidney, 15 (19.7\%) developed end stage renal disease due to tuberculosis, 10 already at clinical presentation and five on follow-up, despite specific treatment. All 57 patients with tuberculosis-related ureteral stenosis lost the corresponding kidney. Fiftynine patients required nephrectomy and two needed polar nephrectomy.

\section{COMMENTS}

Of the urogenital tuberculosis cases described in the literature (range 7-39), the male-female distribution was 2-1, with a mean age of 40.7 years (range 5 to 88 ). In only $36.6 \%$ of the cases was there history or radiographic evidence of previous tuberculosis. In cases where the renal lesions are mainly asymptomatic, and only vesical lesions lead to symptoms (7-14), storage symptoms predominate. A total of $48.5 \%$ of males had scrotal involvement with an epididymis mass, epididymis hardness, or fistula, on physical examination, findings that point to the importance of these signs. Patients in developed countries have 

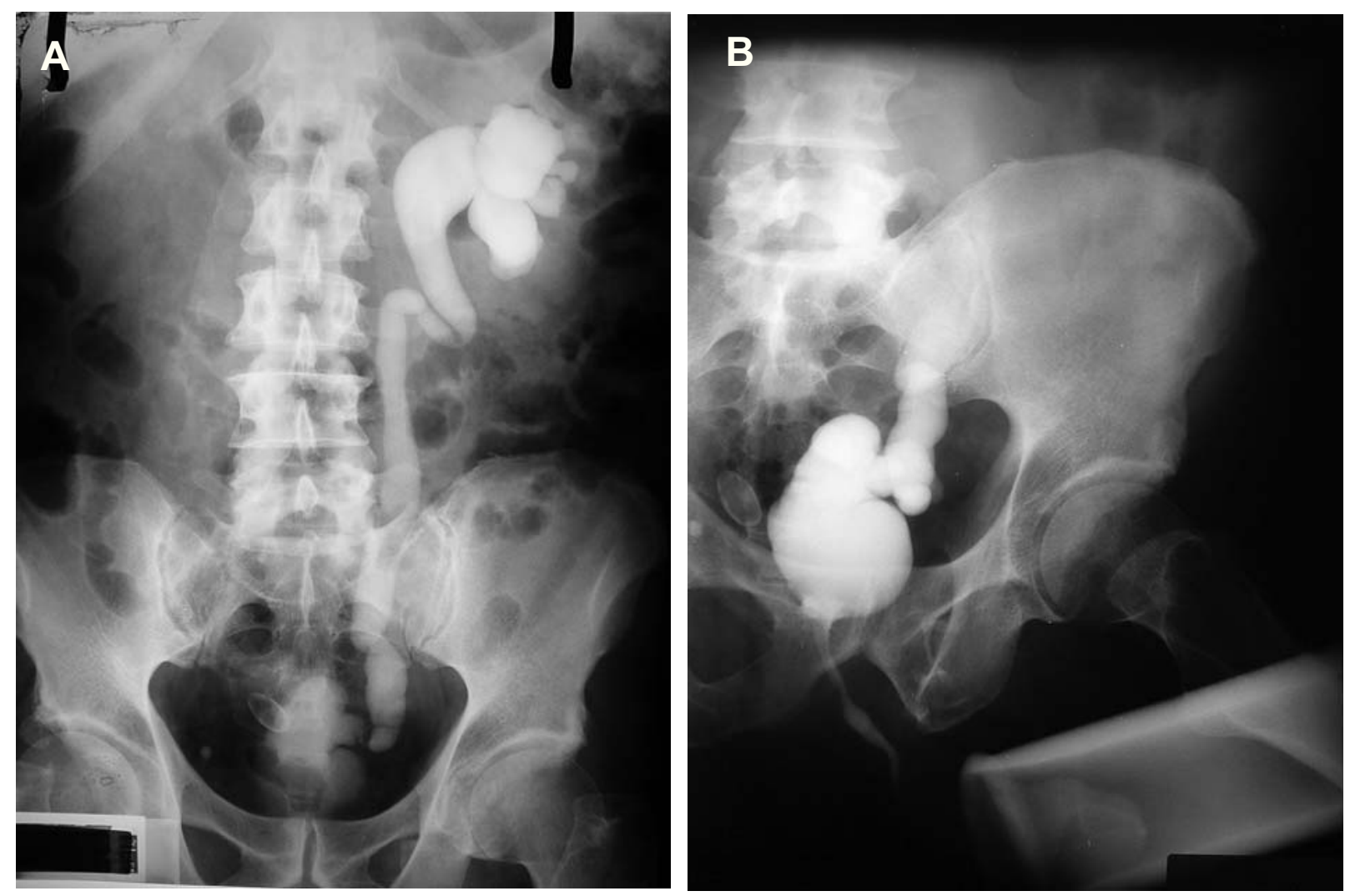

Figure 6 - A) Intravenous urography with right non-function kidney, B) Voiding cystography shows contracted bladder and left vesicoureteral reflux.

fewer specific symptoms and smaller percentages of delayed histopathological diagnoses compared with other countries. As a result, the disease tends to be less serious, with fewer instances of renal failure, unilateral non-function kidney, ablative surgeries, and contracted bladder, with more cases presenting without significant lesions of the upper urinary tract on diagnosis. These data point to a correlation of the timing of the diagnosis with the severity of urogenital tuberculosis. Our findings contrast with those from developed countries, with greater rates of histopathological diagnoses, symptomatic patients at presentation, and severe destruction of the urinary tract $(57.6 \%$ with contracted bladder, $19.7 \%$ with end stage renal disease, $12.5 \%$ with renal failure at initial presentation, and $71.2 \%$ with unilateral renal exclusion in the absence of renal failure or a transplanted kidney). Table-3 shows these features.
Since histopathology provides a delayed urogenital tuberculosis diagnosis, identification of the tuberculosis bacillus in the urine is necessary for early diagnosis. It is achieved through direct smears (Ziehl-Neelsen stain) or through urine culture (Lowestein-Jensen media) $(40,41)$. Direct smears provide a faster result with high specificity (96.7\%) but only 42.1 to $52.1 \%$ sensitivity $(40,41)$. Culture is the diagnostic gold standard for urogenital tuberculosis, however 3 to 6 early morning mid-stream samples are required, the sensitivity varies widely, from $10 \%$ to $90 \%$, and the time to detection of mycobacterium growth may be 4 to 6 weeks (40-42). Faster culture has been achieved with non-radiometric automated or semi-automated liquid culture systems with a 14 to 17 days result (42). Nucleic acid amplification tests, as polymerase chain reaction (PCR), for Mycobacterium tuberculosis identification in the urine may become 
Classification of Urogenital Tuberculosis

Table 3 - Comparison between 3036 cases of urogenital tuberculosis from developed countries (USA, Europe and Japan) with 5925 cases from other countries (Russia, Latin America and Africa) and 80 cases from Brazil.

\begin{tabular}{|c|c|c|c|c|c|}
\hline & Developed & Others & p Value & Brazil & Total \\
\hline Total & 3036 & 5925 & & 80 & 9041 \\
\hline Men & $62.9 \%$ & $65.4 \%$ & 0.02 & $70 \%$ & $65 \%$ \\
\hline Women & $37.1 \%$ & $34.6 \%$ & 0.02 & $30 \%$ & $35 \%$ \\
\hline Median age (years) & 42.6 & 39.2 & & 34 & 40.7 \\
\hline Range (years) & 7 to 88 & 5 to 83 & & 12 to 75 & 5 to 88 \\
\hline Previous tuberculosis & $37.9 \%$ & $38.4 \%$ & 0.66 & $43.8 \%$ & $36.6 \%$ \\
\hline \multicolumn{6}{|l|}{ Signs and symptoms } \\
\hline Storage symptoms & $44.2 \%$ & $55.2 \%$ & $<0.01$ & $72.5 \%$ & $50.7 \%$ \\
\hline Dysuria & $33.8 \%$ & $46.4 \%$ & $<0.01$ & $72.5 \%$ & $38.2 \%$ \\
\hline Lumbar pain & $28.8 \%$ & $42.3 \%$ & $<0.01$ & $28.8 \%$ & $34.4 \%$ \\
\hline Hematuria & $24.5 \%$ & $44.3 \%$ & $<0.01$ & $56.3 \%$ & $35.8 \%$ \\
\hline Epididymis lesion * & $20.6 \%$ & $47.4 \%$ & $<0.01$ & $12.5 \%$ & $48.5 \%$ \\
\hline Fever and malaise & $23.2 \%$ & $19.9 \%$ & 0.28 & $45.0 \%$ & $22.1 \%$ \\
\hline No symptoms & $8.4 \%$ & $0 \%$ & $<0.01$ & $6.3 \%$ & $6.4 \%$ \\
\hline Renal failure & $1.4 \%$ & $10.2 \%$ & $<0.01$ & $19.7 \% * *$ & $5.8 \%$ \\
\hline \multicolumn{6}{|l|}{ Diagnosis } \\
\hline Urine & $79.0 \%$ & $55.4 \%$ & $<0.01$ & $45.0 \%$ & $64.0 \%$ \\
\hline Histopathology & $7.8 \%$ & $38.3 \%$ & $<0.01$ & $31.3 \%$ & $21.8 \%$ \\
\hline $\begin{array}{l}\text { Clinico-radiographic } \\
\text { Kidney }\end{array}$ & $9.6 \%$ & $11.3 \%$ & 0.36 & $23.7 \%$ & $10.5 \%$ \\
\hline Unilateral non-function & $22.8 \%$ & $33.1 \%$ & $<0.01$ & $58.8 \%$ & $27.2 \%$ \\
\hline Normal & $18.8 \%$ & $13.2 \%$ & $<0.01$ & $10.0 \%$ & $15.2 \%$ \\
\hline Contracted bladder & $4.0 \%$ & $11.6 \%$ & $<0.01$ & $58.8 \%$ & $9.3 \%$ \\
\hline Surgeries & $56.6 \%$ & $53.6 \%$ & $<0.01$ & $76.3 \%$ & $55.1 \%$ \\
\hline Ablative & $35.0 \%$ & $26.9 \%$ & $<0.01$ & $76.3 \%$ & $27.6 \%$ \\
\hline Nephrectomy & $27.9 \%$ & $26.0 \%$ & 0.37 & $71.3 \%$ & $28.0 \%$ \\
\hline
\end{tabular}

$p=$ comparison between developed countries (USA, Europe and Japan) and other countries (Latin America, Africa and Russia) through chi-square test. * in relation to male patients; ** in relation to non-transplanted patients.

the ideal diagnostic tool, as it gives results in 24 to 48 hours and allows for the diagnosis to be made even when there are few bacilli $(40,41)$. It has been showed $95.6 \%$ sensitive and $98.1 \%$ specific compared to culture (40) and $94.3 \%$ sensitive and $85.7 \%$ specific compared to bacteriological, histological, or clinicoradiological diagnoses (41).

Primary pulmonary tuberculosis is usually subclinical and self-limited. From the pulmonary focus there is bacillemia and bacilli implants in other organs, renal parenchymatous and prostate colonization ensuing. After six months, spontaneous cicatrisation of the primary pulmonary tuberculosis lesion occurs and the patients enter a latent phase, with 5\% reactivating the disease in the following two years and $5 \%$ in their lifetime. In most cases of active pulmonary or extrapulmonary disease, there is foci reactivation due to a breach of immunity brought about by malnutrition, diabetes mellitus, steroid use, immunosuppressor use, and immunodeficiencies $(3,43)$.

The present urogenital tuberculosis patients' classification in seven groups was based on disease 
physiopathology and made evident the correlation between symptoms and disease's stage. Renal lesions are initially bilateral, cortical, glomerular, and peri-capillary, typical of the hematogenous spread and concomitant with other hematogenous foci in the prostate and other organs beyond the urogenital system $(15,44)$. These foci usually heal, with the patient entering a latent phase. If any immunodeficiency ensues although, miliary tuberculosis with systemic symptoms develops $(3,13)$,from 25 to $62 \%$ of patients with miliary tuberculosis will have a renal lesion with multiple bilateral foci $(9,44)$. In our patients $8.8 \%$ had miliary tuberculosis with bilateral parenchymatous renal lesions and systemic symptoms, characterizing a typical clinical presentation of immune deficient patients. Tuberculosis on a transplanted kidney can be also included in this multiple parenchymatous lesion presentation.

The latent period between pulmonary infection with bacillemia and clinically evident urogenital tuberculosis is 22 years on average, ranging from 1 to 46 years, according to the timing of latent foci (renal, prostate, or epididymis) reactivation (12). Isolated prostate or epididymis foci reactivation may occur, characterizing genital tuberculosis without associated renal lesion. In the reactivation of renal foci, infection progresses from a single unilateral focus, with preservation of the contralateral kidney (15). This explains the greater frequency of unilateral renal tuberculosis $(12,16)$. The contiguous involvement of the collecting system leads to bacilluria and descending spread of the infection to the ureter, bladder, and genital organs $(44,45)$. Thus, a typical clinico-radiographic form exists: unilateral renal tuberculosis with preservation of the contralateral kidney and the bladder. This occurred in $15 \%$ of our cases, with obstruction and dilatation of the collecting system, with invariable renal functional loss. In fact, obstruction of the collecting system (with distal ureteral stenosis as the most frequent finding) is the main cause of renal functional loss in tuberculosis $(11,46,47)$. The focal origin of tuberculosis reactivation is demonstrated by two of our cases with restriction of the disease to the renal pole. If the diagnosis of the urogenital tuberculosis reactivation is at an early stage, we can detect no or minimal radiographic features such as calyceal dilatation due to initial infundibular stricture as we observed in group 2 patients (41).

Another clinico-radiographic presentation was a contracted bladder without renal failure, occurring in $45 \%$ of the patients. There was unilateral nonfunction kidney in practically all cases, with collecting system obstruction and dilatation, primarily $(66.7 \%)$ with unilateral reflux to the function kidney, $27.8 \%$ without reflux, and only $5.5 \%$ with bilateral reflux. All patients with renal failure had bladder contraction and unilateral reflux on investigation. These findings prompted a new hypothesis for the pathophysiology of the urinary tract lesions in urogenital tuberculosis. After reactivation and progression of the unilateral renal focus, the collecting system is implicated, with unilateral descending involvement of the ipsilateral ureter and bladder ensuing (4). Ureteral stenosis with corresponding renal function loss and fibrosis of the bladder wall are the next stages (47). At this stage, the investigations show unilateral non-function kidney with contracted bladder and absence of reflux. Progression of the bladder infection with capacity and compliance reduction leads to distortion of the vesicoureteral junction and vesicoureteral reflux to the functional kidney, the collecting system thus functioning as a buffer for the reduced capacity of the contracted bladder, and with ascending transmission of the high intravesical pressure. Currently, the investigations show unilateral non-function kidney, contracted bladder, and reflux to the function kidney, as seen in most of our patients (Figure-5 and 6). Non-identified and non-treated reflux causes renal lesion due to infection or transmission of intravesical pressure, leading to end stage renal disease. In fact, renal failure patients presented unilateral reflux, underlying the hypothesis that one of the kidneys loses its function because of reflux, and not due to tuberculosis itself. Furthermore, patients with unilateral non-function kidney had ureterohydronephrosis and areas of retraction in the functional kidneys, as well as changes associated with vesicoureteral reflux. The fact that there were no typical tuberculosis lesions in these kidneys, such as ureteral or intra-renal stenosis, underlies the role of reflux nephropathy and not tuberculosis itself, in the pathogenesis of the contralateral involvement.

After the discovery on specific tuberculostatic drugs in the XX century, the profile of urogenital 
tuberculosis experienced dramatic changes, with reduced mortality, cure of initial lesions, reduction in the number of ablative surgeries, and increase in the number of reconstructive surgeries. In the last decades, however, no significant change occurred, in spite of technological advances (48). Since the 60's the importance of an early diagnosis of urogenital tuberculosis has been acknowledged as the main step towards renal preservation (47). A systematic search for urogenital tuberculosis in patients with pulmonary disease yielded $10 \%$ of positive cultures (66.7\% asymptomatic and 58\% with normal urine exam and absence of renal lesions on pyelography) (49). In the other two series, where cultures were routinely grown, a greater frequency of asymptomatic patients without lesions on intravenous pyelography was found $(8,50)$. Although bacilluria is invariably associated with renal lesion, detection of pre-clinical bacilluria allows for an early diagnosis to be made, at a moment when the lesions are amenable to cure and the development of severe and destructive lesions can be avoided (45). Of our patients with minimum or absent lesions, two had no urologic symptoms and the others had isolated hematuria. Thus, a systematic search for urogenital tuberculosis, regardless of symptoms, is warranted for early case detection. We propose that all patients with macroscopic hematuria or persisting microscopic hematuria or leucocituria should be submitted to Mycobacterium tuberculosis urinary culture or PCR analyses. We also propose yearly urinalysis for microscopic hematuria or leucocituria detection in patients with pulmonary tuberculosis history or immunological impairment. However, the better definition of higher risk groups and optimal diagnosis strategy warrants further studies.

\section{CONFLICT OF INTEREST}

None declared.

\section{REFERENCES}

1. Brasil - Ministério da Saúde, Guia de Vigilância Epidemiológica, Brasília. 1998; Cap. 5.34: pp.1-13. Tuberculose.
2. Dias Filho AC, Netto Júnior NR: Aspectos atuais da tuberculose urinária e genital masculina. Jornal Brasileiro de Urologia. 1999; 25: 453-65.

3. Leite OH: Tuberculosis. Problems in General Surgery. 2001; 18: 69-78.

4. Gow JG: Genitourinary tuberculosis. In: Walsh PC, Retik AB, Vaughan ED, Wein AJ. Campbell's Urology. ( $7^{\mathrm{a}}$ ed.), Philadelphia, WB Saunders Company. 1998; Vol. 1: pp 807-36.

5. Hijjar MA, Oliveira MJPR, Teixeira GM: A tuberculose no Brasil e no mundo. Boletim de Pneumologia Sanitária. 2001; 9: 9-16.

6. Schubert GE, Haltaufderheide T, Golz R: Frequency of urogenital tuberculosis in an unselected autopsy series from 1928 to 1949 and 1976 to 1989. Eur Urol. 1992; 21: 216-23.

7. Flechner SM, Gow JG: Role of nephrectomy in the treatment of non-functioning or very poorly functioning unilateral tuberculous kidney. J Urol. 1980; 123: 822-5.

8. Ferrie BG, Rundle JS: Genito-urinary tuberculosis in Glasgow 1970 to 1979: a review of 230 patients. Scott Med J. 1985; 30: 30-4.

9. Simon HB, Weinstein AJ, Pasternak MS, Swartz MN, Kunz LJ: Genitourinary tuberculosis. Clinical features in a general hospital population. Am J Med. 1977; 63: 410-20.

10. Nzerue C, Drayton J, Oster R, Hewan-Lowe K: Genitourinary tuberculosis in patients with HIV infection: clinical features in an inner-city hospital population. Am J Med Sci. 2000; 320: 299-303.

11. Ramanathan R, Kumar A, Kapoor R, Bhandari M: Relief of urinary tract obstruction in tuberculosis to improve renal function. Analysis of predictive factors. Br J Urol. 1998; 81: 199-205.

12. Christensen WI: Genitourinary tuberculosis: review of 102 cases. Medicine (Baltimore). 1974; 53: 377-90.

13. Alvarez S, McCabe WR: Extrapulmonary tuberculosis revisited: a review of experience at Boston City and other hospitals. Medicine (Baltimore). 1984; 63: 2555.

14. Gokalp A, Gultekin EY, Ozdamar S: Genito-urinary tuberculosis: a review of 83 cases. Br J Clin Pract. 1990; 44: 599-600.

15. Narayana A: Overview of renal tuberculosis. Urology. 1982; 19: 231-7.

16. García-Rodríguez JA, García Sánchez JE, Muñoz Bellido JL, Montes Martínez I, Rodríguez Hernández J, Fernández Gorostarzu J, et al.: Genitourinary tuberculosis in Spain: review of 81 cases. Clin Infect Dis. 1994; 18: 557-61. 
17. Mochalova TP, Starikov IY: Reconstructive surgery for treatment of urogenital tuberculosis: 30 years of observation. World J Surg. 1997; 21: 511-5.

18. Kao SC, Fang JT, Tsai CJ, Chen KS, Huang CC: Urinary tract tuberculosis: a 10-year experience. Changgeng Yi Xue Za Zhi. 1996; 19: 1-9.

19. Caro Muñoz M, Corvalán JR, Tenório JP, Vergara JF: Diagnosis of urogenital tuberculosis: a retrospective review (1974 - 1986). Bol Hosp San Juan de Díos. 1988; 35(4): 223-6.

20. Venegas MP, Corti OD, Foneron BA: Genitourinary TBC: experience of Hospital de Valdiva 1979-1984 years. Rev Chil Urol. 1990; 53: 131-4.

21. Núñez Vásquez N, Susaeta S R, Vogel M, Olate C: Genitourinay tuberculosis study: urologic service, Hospital San Juan de Dios 1975-1989. Rev Chil Urol. 1990; 53: 135-7.

22. Allen FJ, de Kock ML: Genito-urinary tuberculosis-experience with 52 urology inpatients. S Afr Med J. 1993; 83: 903-7.

23. Bennani S, Hafiani M, Debbagh A, el Mrini M, Benjelloun S: Urogenital tuberculosis. Diagnostic aspects. J Urol (Paris). 1995; 101: 187-90.

24. Hinostroza Fuschlocher JÁ, Gorena Palominos M, Pastor Arroyo P, Rivera P, Venegas P, Orío M, Peldoza $\mathrm{M}$, et al.: Genitourinary tuberculosis at Araucanía: experience at Hospital Regional de Temuco. Rev Chil Urol. 1998; 63: 113-7.

25. Mnif A, Loussaief H, Ben Hassine L, Chebil M, Ayed M. Aspects of evolving urogenital tuberculosis. 60 cases. Ann Urol (Paris). 1998; 32: 283-9.

26. Lopez de Mesa B, Cruz A, Gomes P: Review of patients with genitourinary tuberculosis in San Juan de Dios Hospital. Urol Colomb. 1998; 7: 43-52.

27. Benchekroun A, Lachkar A, Soumana A, Farih MH, Belahnech Z, Marzouk M, et al.: Urogenital tuberculosis. 80 cases. Ann Urol (Paris). 1998; 32: 89-94.

28. Chtourou M, Kbaier I, Attyaoui F, Nouira Y, Barrak A, Sayari S, et al.: Management of genito-urinary tuberculosis. A report of 225 cases. J Urol. 1999; 161 (Suppl 4): Abstract 34.

29. Buchholz NP, Salahuddin S, Haque R: Genitourinary tuberculosis: a profile of 55 in-patients. J Pak Med Assoc. 2000; 50: 265-9.

30. Hemal AK, Gupta NP, Kumar R: Comparison of retroperitoneoscopic nephrectomy with open surgery for tuberculous nonfunctioning kidneys. J Urol. 2000; 164: 32-5.

31. el Khader K, Lrhorfi MH, el Fassi J, Tazi K, Hachimi M, Lakrissa A. Urogenital tuberculosis. Experience in 10 years. Prog Urol. 2001; 11: 62-7.
32. Gokce G, Kilicarslan H, Ayan S, Tas F, Akar R, Kaya K, et al.: Genitourinary tuberculosis: a review of 174 cases. Scand J Infect Dis. 2002; 34: 338-40.

33. Barrios F, Martinez-Peschard L, Rosas A: Surgical management of tuberculosis. BJU Int. 2002; 90 (Suppl 2): $\mathrm{p} 26$.

34. Ishibashi Y, Takeda T, Nishimura R, Ohshima H: A clinical observation of genitourinary tract tuberculosis during the last decade. Hinyokika Kiyo. 1985; 31: 107-12.

35. Gow JG: Genitourinary tuberculosis: a 7-year review. Br J Urol. 1979; 51: 239-44.

36. Gow JG, Barbosa S: Genitourinary tuberculosis. A study of 1117 cases over a period of 34 years. Br J Urol. 1984; 56: 449-55.

37. Fuse H, Imazu A, Shimazaki J. A clinical observation on urogenital tuberculosis. Hinyokika Kiyo. 1984; 30: 299-304.

38. Fischer M, Flamm J: The value of surgical therapy in the treatment of urogenital tuberculosis. Urologe A. 1990; 29: 261-4.

39. Zwergel U, Wullich B, Rohde V, Zwergel T: Surgical management of urinary tuberculosis: a review of 341 patients. J Urol. 1999; 161 (Suppl 4): Abstract 33.

40. Moussa OM, Eraky I, El-Far MA, Osman HG, Ghoneim MA: Rapid diagnosis of genitourinary tuberculosis by polymerase chain reaction and non-radioactive DNA hybridization. J Urol. 2000; 164: 584-8.

41. Hemal AK, Gupta NP, Rajeev TP, Kumar R, Dar L, Seth P: Polymerase chain reaction in clinically suspected genitourinary tuberculosis: comparison with intravenous urography, bladder biopsy, and urine acid fast bacilli culture. Urology. 2000; 56: 570-4.

42. Dinnes J, Deeks J, Kunst H, Gibson A, Cummins E, Waugh N, et al.: A systematic review of rapid diagnostic tests for detection of tuberculosis infection. Health Technology Assessment. 2007; 11: 1-196.

43. Cos LR, Cockett AT: Genitourinary tuberculosis revisited. Urology. 1982; 20: 111-7.

44. Medlar EM, Spain DM, Holliday RW: Post-mortem compared with clinical diagnosis of genito-urinary tuberculosis in adult males. J Urol. 1949; 61: 1078-88.

45. Medlar EM, Sasano KT: Experimental renal tuberculosis, with special reference to excretory bacilluria. Am Rev Tuberc. 1924; 10: 370-7.

46. Barrie HJ, Kerr WK, Gale GL: The incidence and pathogenesis of tuberculous strictures of the renal pyelus. J Urol. 1967; 98: 584-9.

47. Kerr WK, Gale GL, Peterson KS: Reconstructive surgery for genitourinary tuberculosis. J Urol. 1969; 101: 254-66. 


\section{Classification of Urogenital Tuberculosis}

48. Ross JC: Renal tuberculosis. Br J Urol. 1953; 25: 277315 .

49. Bentz RR, Dimcheff DG, Nemiroff MJ, Tsang A, Weg JG: The incidence of urine cultures positive for Mycobacterium tuberculosis in a general tuberculosis patient population. Am Rev Respir Dis. 1975; 111: $647-50$.

\section{Correspondence address:}

Dr. André Avarese de Figueiredo

Rua Irineu Marinho 365 / 801, Bloco 3

Juiz de Fora, MG, 36021-580, Brazil

Fax: + $55113287-6922$

E-mail: andreavaresef@gmail.com

\section{EDITORIAL COMMENTS}

On May 4, 1953, The New York Times published a report from The World Health Organization that stated "there has been an "extraordinary" drop in mortality from tuberculosis of the respiratory system since the end of World War II. The development of anti-tuberculosis medication, improved living standards and Public Health measures made tuberculosis an almost forgotten disease particularly in developed countries. Unfortunately, this has not been the case. As noted in the paper tuberculosis continues to be a major world wide public health problem. Poor socio-
50. Butler MR, O'Flynn JD: Reactivation of genito-urinary tuberculosis: a retrospective review of 838 cases. Eur Urol. 1975; 1: 14-7.

Accepted after revision:

March 17, 2008

economic conditions, immune suppression, AIDS are factors that perpetuate tuberculosis. The authors noted that 2 to $20 \%$ of patients with pulmonary can manifest urogenital tuberculosis. Their paper 'classified' GU tuberculosis into 7 categories including one category with 'No or minimal' disease.

This paper renews a known caveat for the Urologist. 'Atypical' urologic manifestations such as renal scaring, scrotal masses, idiopathic pyuria or hematuria mandate an assessment for tuberculosis.

Dr. G. J. Wise Division of Urology Maimonides Medical Center Brooklyn, New York, USA E-mail:gwise@maimonidesmed.org 